\title{
Multiuser Detection for the Uplink of Prefix-Assisted DS-CDMA Systems Employing Multiple Transmit and Receive Antennas
}

\author{
Paulo Silva ${ }^{(1)}$ and Rui Dinis ${ }^{(2)}$ \\ ${ }^{(1)}$ EST, Univ. of Algarve, Portugal, psilva@ualg.pt \\ ${ }^{(2)}$ ISR-IST, Tech. Univ. of Lisbon, Portugal, rdinis@ist.utl.pt
}

\begin{abstract}
To achieve high spectral and power efficiencies, future broadband wireless systems should combine spatial multiplexing techniques employing multiple transmit and receive antennas with transmission schemes suitable to severely time-dispersive channels such as block transmission techniques, with appropriate CP (Cyclic Prefix) and employing FDE (Frequency-Domain Equalization). Moreover, the signal to be transmitted by the MT (Mobile Terminal) should have a reduced peak power, as well as low envelope fluctuations, so as to allow an efficient power amplification.

In this paper we consider the uplink transmission within a DS-CDMA system employing CP-assisted block transmission techniques combined with spatial multiplexing techniques that require multiple antennas at both the transmitter and the receiver. We present an efficient frequency-domain receiver structure with an iterative MUD (MultiUser Detection).

It is shown that the performance of the proposed receiver can be close to the single-user MFB (Matched Filter Bound), even for fully loaded systems, in severely time-dispersive channels and/or in the presence of strong interfering signals. ${ }^{1}$
\end{abstract}

\section{Introduction}

The design of future broadband wireless systems presents a big challenge, since these systems should be able to cope with severely time-dispersive channels and are expected to have high spectral and power efficiencies. Moreover, a lowcost and efficient power amplification is recommendable at the MT (Mobile Terminal).

Block transmission techniques, with appropriate CP (Cyclic Prefix) and employing FDE (Frequency-Domain Equalization), are known to be suitable for high data rate transmission over severe time-dispersive channels, since they allow lowcomplexity, FFT-based (Fast Fourier Transform) implementations [4], [5]. On the other hand, by using multiple antennas

${ }^{1}$ This work was partially supported by the FCT project POSI/CPS/46701/2002 - MC-CDMA and the FCT/POCI 2010 research grant SFRH / BD / 24520 / 2005. at both the transmitter and receiver, we can increase significantly spectral efficiencies of wireless communication systems, namely through the use of spatial multiplexing techniques [1].

Therefore, future broadband wireless systems are expected to combine $\mathrm{CP}$-assisted block transmission techniques with multiantenna schemes. These techniques can be combined with either multicarrier modulations, such as the OFDM schemes (Orthogonal Frequency Division Multiplexing) [2], or singlecarrier modulations [3]. Due to the lower envelope fluctuations, the later ones are preferable for the uplink transmission [4], [5] (i.e., the transmission form the MT (Mobile Terminal) to the BS (Base Station)).

DS-CDMA schemes (Direct Sequence Code Division Multiple Access) [6] are especially interesting for cellular systems, due to their good capacities and high system flexibility. Moreover, contrarily to TDMA schemes (Time Division Multiple Access), all users transmit continuously, regardless of the bit rates, reducing significantly the peak power requirements for the amplifiers. Moreover, since DS-CDMA schemes can be regarded as single-carrier modulations, the transmitted signal associated to each spreading code can have low envelope fluctuations. Therefore, DS-CDMA schemes are good candidates for broadband wireless systems, especially at the uplink.

DS-CDMA schemes can be combined with CP-assisted block transmission techniques, allowing a frequency-domain receiver design that have relatively low complexity, even for severely time-dispersive channels. The receiver is particularly simple at the downlink: since all spreading codes are affected by the same multipath channel, the receiver can be based on a linear FDE (Frequency-Domain Equalizer), operating at the chip level, followed by the despreading procedure [7]. The performances can be further improved if the linear FDE is replaced by a more powerful IB-DFE (Iterative Block Decision feedback Equalization) [8], [9], especially for fully loaded scenarios and/or in the presence of strong interference levels [10].

In this paper we consider the uplink transmission within a DS-CDMA system employing CP-assisted block transmission techniques combined with spatial multiplexing techniques, requiring multiple antennas at both the transmitter and the receiver. We present an efficient frequency-domain receiver structure with an iterative MUD (MultiUser Detection) that 
combines IB-DFE principles with interference cancelation techniques.

This paper is organized as follows: The CP-assisted DSCDMA schemes with spatial multiplexing considered here are described in sec. II. In sec. III we describe the iterative, frequency-domain receiver adopted in this paper. Some practical implementations issues are discussed in sec. IV. Sec. V presents a set of performance results and sec. VI is concerned with the conclusions of the paper.

\section{System Characterization}

In this paper we consider the uplink transmission in DSCDMA systems employing CP-assisted block transmission techniques. We have a spreading factor $K$ and $P$ MTs. As depicted in fig. 1 , the BS has $L_{R}$ receive antennas and the $p$ th MT has $L_{T}^{(p)}$ transmit antennas, each one transmitting a different stream of data symbols. It is assumed that the received blocks associated to each MT are synchronized in time (in practice, this means that there is a suitable "time-advance" mechanism allowing perfect synchronization, although just a coarse synchronization is required since some time misalignments can be absorbed by the $\mathrm{CP}$ ).

\section{(A)}

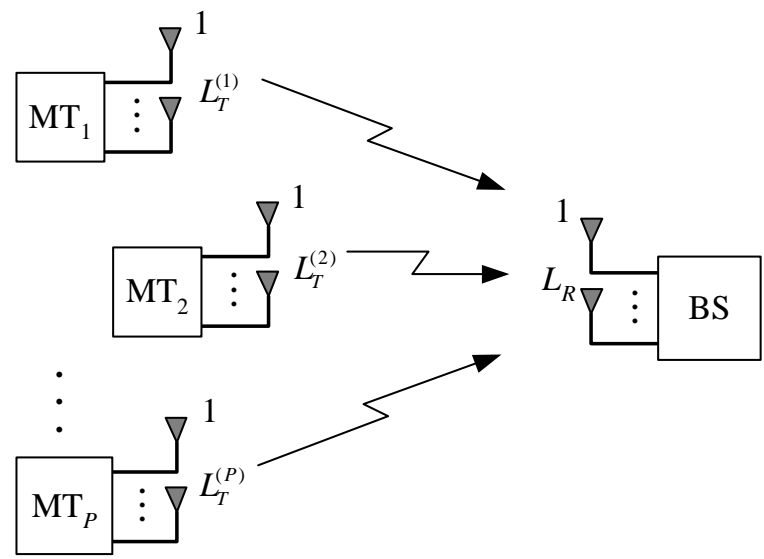

(B)

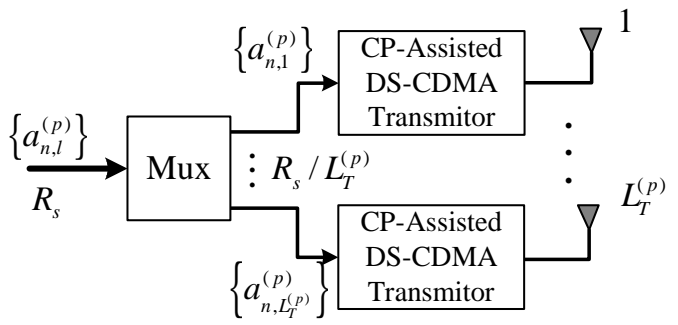

Fig. 1. System characterization (A) and detail of the $p$ th MT (B), with spatial multiplexing of degree $L_{T}^{(p)}$ and data rate $R_{s}$.

The size- $M$ data block to be transmitted by the $l$ th antenna of the $p$ th MT is $\left\{a_{n, l}^{(p)} ; n=0,1, \ldots, M-1\right\}$, with $a_{n, l}^{(p)}$ selected from a given constellation. The corresponding chip block to be transmitted is $\left\{s_{n, l}^{(p)} ; n=0,1, \ldots, N-1\right\}$, where $N=$ $M K$ and $s_{n, l}^{(p)}=a_{\lfloor n / K\rfloor, l}^{(p)} c_{n, l}^{(p)}(\lfloor x\rfloor$ denotes "larger integer not higher than $x$ "), with $c_{n, l}^{(p)}$ denoting the spreading symbols ${ }^{2}$. The spreading sequence is assumed to be periodic, with period $K$ (i.e., $c_{n+K, l}^{(p)}=c_{n, l}^{(p)}$ ).

The received signal associated to the $r$ th antenna of the BS is sampled at the chip rate (the generalization for multiple samples per chip is straightforward) and the $\mathrm{CP}$ is removed, leading to the time-domain block $\left\{y_{n}^{(r)} ; n=0,1, \ldots, N-1\right\}$. It can be shown that, when the $\mathrm{CP}$ is longer than the overall channel impulse response, the corresponding frequency-domain block is $\left\{Y_{k}^{(r)} ; k=0,1, \ldots, N-1\right\}$, where

$$
Y_{k}^{(r)}=\sum_{p=1}^{P} \sum_{l=1}^{L_{T}^{(p)}} S_{k, l}^{(p)} \xi_{l}^{(p)} H_{k, l}^{C h, p, r}+N_{k}^{(r)}
$$

with $H_{k, l}^{C h, p, r}$ denoting the channel frequency response between the $l$ th transmit antenna of the $p$ th MT and the $r$ th receive antenna of the BS, at the $k$ th frequency (without loss of generality, it is assumed that $\left.E\left[\left|H_{k, l}^{C h, p, r}\right|^{2}\right]=1\right) . N_{k}$ is the channel noise at the $r$ th receive antenna, for the $k$ th frequency and $\xi_{l}^{(p)}$ is a scale factor that accounts for the combined effect of the propagation losses and the power assigned to the $l$ th antenna of the $p$ th MT. The frequency-domain block $\left\{S_{k, l}^{(p)} ; k=0,1, \ldots, N-1\right\}$ is the DFT of the chip block transmitted by the $l$ th antenna of the $p$ th $\mathrm{MT},\left\{s_{n, l}^{(p)} ; n=\right.$ $0,1, \ldots, N-1\}$. Since $s_{n, l}^{(p)}=a_{\lfloor n / K\rfloor, l}^{(p)} c_{n, l}^{(p)}$, it can be easily shown that

$$
S_{k, l}^{(p)}=A_{k, l}^{\prime(p)} C_{k, l}^{\prime(p)}
$$

where $\left\{A_{k, l}^{\prime(p)} ; k=0,1, \ldots, N-1\right\}=\operatorname{DFT}\left\{a_{n, l}^{\prime(p)} ; n=\right.$ $0,1, \ldots, N-1\}$, with

$$
a_{n, l}^{\prime(p)}= \begin{cases}a_{n^{\prime}, l}^{(p)}, & n=n^{\prime} K \\ 0, & \text { otherwise }\end{cases}
$$

and $\left\{C_{k, l}^{\prime(p)} ; k=0,1, \ldots, N-1\right\}=\operatorname{DFT}\left\{c_{n, l}^{\prime(p)} ; n=\right.$ $0,1, \ldots, N-1\}$, with

$$
c_{n, l}^{\prime(p)}= \begin{cases}c_{n, l}^{(p)}, & 0 \leq n<K \\ 0, & \text { otherwise. }\end{cases}
$$

Clearly, $A_{k, l}^{\prime(p)}=\frac{1}{K} A_{k \bmod M, l}^{(p)}, k=0,1, \ldots, N-1$, with $\left\{A_{k, l}^{(p)} ; k=0,1, \ldots, M-1\right\}=\mathrm{DFT}\left\{a_{n, l}^{(p)} ; n=0,1, \ldots, M-\right.$ $1\}$. This means that, apart a constant, the block $\left\{A_{k, l}^{\prime(p)} ; k=\right.$ $0,1, \ldots, N-1\}$ is the size- $N$ periodic extension of the DFT of the data block associated to the lth antenna of the $p$ th MT $\left\{A_{k, l}^{(p)} ; k=0,1, \ldots, M-1\right\}$. This multiplicity in the $A_{k, l}^{\prime}(p)$ is related to the spectral correlations that are inherent to the cyclostationary nature of the transmitted signals [11]. Therefore,

$$
Y_{k}^{(r)}=\sum_{p=1}^{P} \sum_{l=1}^{L_{T}^{(p)}} A_{k, l}^{(p)} H_{k, l}^{(p, r)}+N_{k}^{(r)}
$$

${ }^{2}$ It will be shown in the following that the different transmit antennas associated to a given MT might have the same spreading code or not. 
with

$$
H_{k, l}^{(p, r)}=\frac{1}{K} \xi_{l}^{(p)} H_{k, l}^{C h, p, r} C_{k, l}^{\prime(p)}
$$

denoting the equivalent channel frequency response between the $l$ th transmit antenna of the $p$ th MT and the $r$ th receive antenna of the BS, for the $k$ th frequency. (5) is equivalent to

$$
\mathbf{Y}_{k}=\mathbf{H}_{k}^{T} \mathbf{A}_{k}+\mathbf{N}_{k}
$$

$\left((\cdot)^{T}\right.$ denote the transpose matrix), with $\mathbf{Y}_{k}$ $\left[\begin{array}{lll}Y_{k}^{(1)} \cdots Y_{k}^{\left(L_{R}\right)} & \cdots & Y_{k+(K-1) M}^{(1)} \cdots Y_{k+(K-1) M}^{\left(L_{R}\right)}\end{array}\right]^{T}$ $\mathbf{N}_{k}=\left[\begin{array}{llll}N_{k}^{(1)} \cdots N_{k}^{\left(L_{R}\right)} & \cdots & N_{k+(K-1) M}^{(1)} \cdots N_{k+(K-1) M}^{\left(L_{R}\right)}\end{array}\right]^{T}$, $\mathbf{A}_{k}=\left[A_{k, 1}^{(1)} \cdots A_{k, L_{T}^{(1)}}^{(1)} \cdots A_{k, 1}^{(P)} \cdots A_{k, L_{T}^{(P)}}^{(P)}\right]^{T}$ and

$$
\mathbf{H}_{k}=\left[\begin{array}{ccc}
\mathbf{H}_{0}^{(1)} & \cdots & \mathbf{H}_{K-1}^{(1)} \\
\vdots & & \vdots \\
\mathbf{H}_{0}^{(P)} & \cdots & \mathbf{H}_{K-1}^{(P)}
\end{array}\right]
$$

with

$$
\mathbf{H}_{q}^{(p)}=\left[\begin{array}{ccc}
H_{k+q M, 1}^{(p, 1)} & \cdots & H_{k+q M, 1}^{\left(p, L_{R}\right)} \\
\vdots & & \vdots \\
H_{k+q M, L_{T}^{(P)}}^{(p, 1)} & \cdots & H_{k+q M, L_{T}^{(P)}}^{\left(p, L_{R}\right)}
\end{array}\right] .
$$

Since we have $K \cdot L_{R}$ replicas associated to each $A_{k, l}^{(p)}$ we can separate $K \cdot L_{R}$ different transmitted layers at the BS, i.e., we should have

$$
N_{L}=\sum_{p=1}^{P} L_{T}^{(p)} \leq K L_{R}
$$

for an ideal separation, with $N_{L}$ denoting the total number of transmitted layers ${ }^{3}$.

\section{Receiver Design}

We consider an iterative frequency-domain MUD receiver that combines IB-DFE principles with interference cancelation. Each iteration consists of $N_{L}$ detection stages, one for each of the different layers. When detecting a given layer, the interference from the other layers is canceled, as well as the residual ISI associated to that layer. These interference and residual ISI cancelations take into account the reliability of each of the previously detected layers.

For a given iteration, the detection of the $l$ th layer of the $p$ th MT employs the structure depicted in fig. 2, where we have $L_{R}$ feedforward filters (one for each receive antenna), followed by a decimation procedure and $N_{L}$ feedback filters (one for each layer). The feedforward filters are designed to minimize both the ISI and the interference that cannot be canceled by the feedback filters, due to decision errors in the previous detection steps. After an IDFT operation, the corresponding time-domain outputs are passed through a hard-decision device so as to

\footnotetext{
${ }^{3}$ For an overloaded system, (10) does not hold. However, it should be noted that our receiver might still be able to separate the layers in slightly overloaded systems, although with some performance degradation.
}

provide an estimate of the data block transmitted by that layer. For the case where we do not have any information about the users' data blocks, the receiver reduces to a linear frequencydomain MUD.

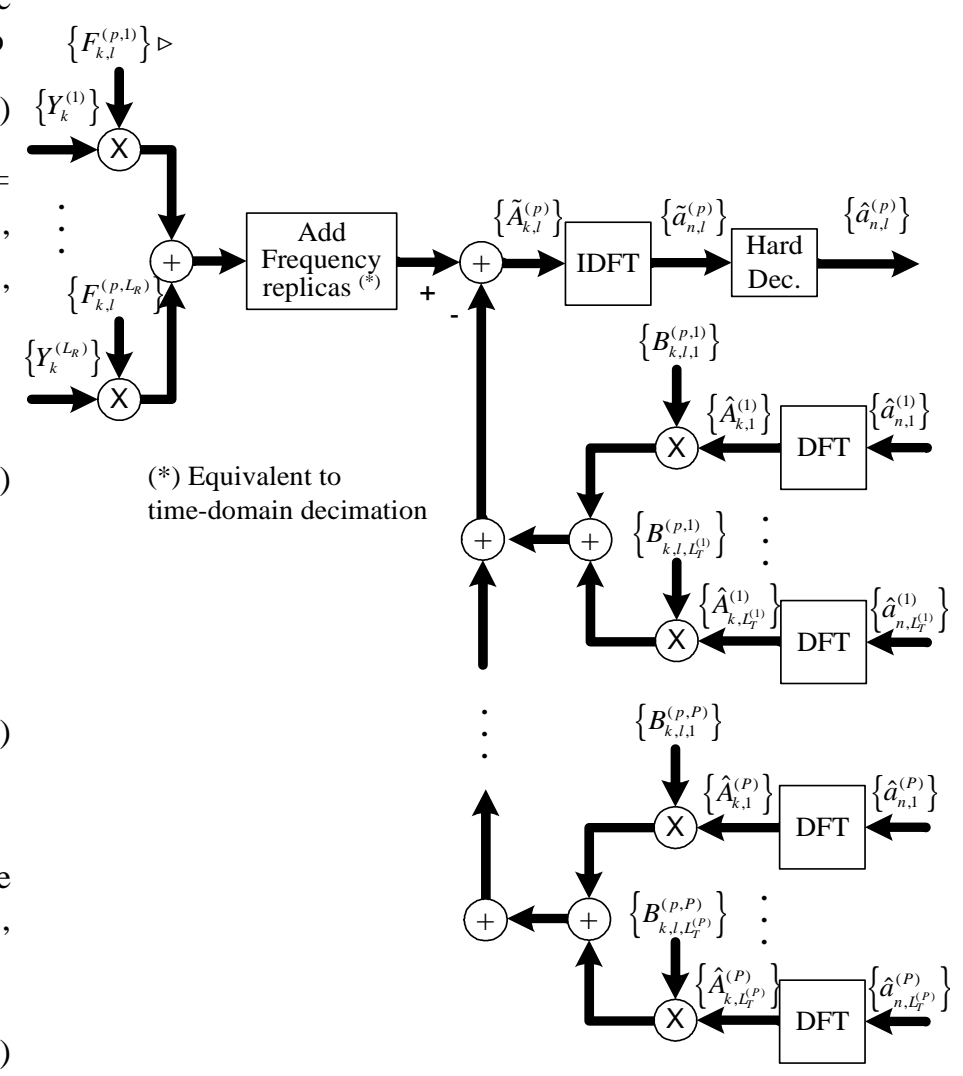

Fig. 2. Detection of the $l$ th layer of the $p$ th MT.

For each iteration, the frequency-domain samples associated with the $l$ th layer of the $p$ th MT at the detector output are given by

$$
\tilde{A}_{k, l}^{(p)}=\sum_{r=1}^{L_{R}} \sum_{q=0}^{K-1} F_{k+q M, l}^{(p, r)} Y_{k+q M}^{(r)}-\sum_{p^{\prime}=1}^{P} \sum_{l^{\prime}=1}^{L_{T}^{\left(p^{\prime}\right)}} B_{k, l, l^{\prime}}^{\left(p, p^{\prime}\right)} \hat{A}_{k, l^{\prime}}^{\left(p^{\prime}\right)}
$$

where $\left\{F_{k, l}^{(p, r)} ; k=0,1, \ldots, N-1 ; r=1,2, \ldots, L_{R}\right\}$ denote the feedforward coefficients and $\left\{B_{k, l, l^{\prime}}^{\left(p, p^{\prime}\right)} ; k=0,1, \ldots, M-\right.$ $\left.1 ; p^{\prime}=1,2, \ldots, P ; l^{\prime}=1,2, \ldots, L_{T}^{\left(p^{\prime}\right)}\right\}$ denote the feedback coefficients. The coefficients $\left\{B_{k, l, l}^{(p, p)} ; k=0,1, \ldots, M-1\right\}$ are used for residual ISI cancellation and the coefficients $\left\{B_{k, l, l^{\prime}}^{\left(p, p^{\prime}\right)} ; k=0,1, \ldots, M-1\right\}\left(l^{\prime} \neq l\right.$ or $\left.p \neq p^{\prime}\right)$ are used for interference cancelation. The block $\left\{\hat{A}_{k, l^{\prime}}^{\left(p^{\prime}\right)} ; k=0,1, \ldots, M-\right.$ $1\}$ is the DFT of the block $\left\{\hat{a}_{n, l^{\prime}}^{\left(p^{\prime}\right)} ; n=0,1, \ldots, M-1\right\}$, where the time-domain samples $\hat{a}_{n, l^{\prime}}^{\left(p^{\prime}\right)}, n=0,1, \ldots, M-1$, are the latest estimates for the transmitted symbols of the $l^{\prime}$ th layer of the $p^{\prime}$ th MT, i.e., the hard-decisions associated with the block of time-domain samples $\left\{\tilde{a}_{n, l^{\prime}}^{\left(p^{\prime}\right)} ; n=0,1, \ldots, M-1\right\}$ $=\operatorname{IDFT}\left\{\tilde{A}_{k, l^{\prime}}^{\left(p^{\prime}\right)} ; k=0,1, \ldots, M-1\right\}$. For the $i$ th iteration 
$\hat{a}_{n, l^{\prime}}^{\left(p^{\prime}\right)}$ is associated with the $i$ th iteration for $l^{\prime}<l$ and with the $(i-1)$ th iteration for $l^{\prime} \geq l$ (in the first iteration, we do not have any information for $l^{\prime} \geq l$ and $\hat{a}_{n, l^{\prime}}^{\left(p^{\prime}\right)}=0$ ).

Using matrix notation, (11) can be rewritten in the form

$$
\tilde{A}_{k, l}^{(p)}=\mathbf{F}_{k, l}^{(p) T} \mathbf{Y}_{k}-\mathbf{B}_{k, l}^{(p) T} \hat{\mathbf{A}}_{k}
$$

where the vector $\hat{\mathbf{A}}_{k}$ is defined as $\mathbf{A}_{k}, \quad \mathbf{F}_{k, l}^{(p)}=$ $\left[F_{k, l}^{(p, 1)} \cdots F_{k, l}^{\left(p, L_{R}\right)} \quad \cdots \quad F_{k+(K-1) M, l}^{(p, 1)} \cdots F_{k+(K-1) M, l}^{\left(p, L_{R}\right)}\right]^{T}$, and $\mathbf{B}_{k, l}^{(p)}=\left[B_{k, l, 1}^{(p, 1)} \cdots B_{k, l, L_{T}^{(1)}}^{(p, 1)} \cdots B_{k, l, 1}^{(p, P)} \cdots B_{k, l, L_{T}^{(P)}}^{(p, P)}\right]^{T}$.

Due to decision errors, we have $\hat{a}_{n, l}^{(p)} \neq a_{n, l}^{(p)}$ for some symbols. Consequently, $\hat{A}_{k, l}^{(p)} \neq A_{k, l}^{(p)}$. For the computation of the receiver coefficients, it is assumed that

$$
\hat{A}_{k, l}^{(p)}=\rho_{l}^{(p)} A_{k, l}^{(p)}+\Delta_{k, l}^{(p)}
$$

where $E\left[\Delta_{k, l}^{(p)}\right] \approx 0, E\left[\Delta_{k, l}^{(p)} A_{k^{\prime}, l}^{(p)}\right] \approx 0$, regardless of $k$ and $k^{\prime}$, and $E\left[\left|\Delta_{k, l}^{(p)}\right|^{2}\right]=\left(1-\rho_{l}^{(p) 2}\right) E\left[\left|A_{k, l}^{(p)}\right|^{2}\right]$. The correlation coefficient $\rho_{l}^{(p)}$ is defined as

$$
\rho_{l}^{(p)}=\frac{E\left[\hat{a}_{n, l}^{(p)} a_{n, l}^{(p) *}\right]}{E\left[\left|a_{n, l}^{(p)}\right|^{2}\right]}=\frac{E\left[\hat{A}_{k, l}^{(p)} A_{k, l}^{(p) *}\right]}{E\left[\left|A_{k, l}^{(p)}\right|^{2}\right]} .
$$

and can be regarded as the blockwise reliability of the estimates $\hat{a}_{n, l}^{(p)}$.

In matrix notation, (13) takes the form

$$
\hat{\mathbf{A}}_{k}=\mathbf{P A}_{k}+\Delta_{k}
$$

with $\Delta_{k}=\left[\Delta_{k, 1}^{(1)} \cdots \Delta_{k, L_{T}^{(1)}}^{(1)} \cdots \Delta_{k, 1}^{(P)} \cdots \Delta_{k, L_{T}^{(P)}}^{(P)}\right]^{T}$ and

$$
\mathbf{P}=\left[\begin{array}{ccc}
\mathbf{P}^{(1)} & \cdots & 0 \\
& \ddots & \\
0 & \cdots & \mathbf{P}^{(P)}
\end{array}\right]
$$

where

$$
\mathbf{P}^{(p)}=\left[\begin{array}{ccc}
\rho_{1}^{(p)} & \cdots & 0 \\
& \ddots & \\
0 & \cdots & \rho_{L_{T}^{(p)}}^{(p)}
\end{array}\right] .
$$

It can be shown that the optimum feedforward coefficients in the MMSE sense can be written as

$$
\mathbf{F}_{k, l}^{(p)}=\frac{1}{\gamma_{l}^{(p)}}\left[\mathbf{H}_{k}^{H}\left(\mathbf{I}-\mathbf{P}^{2}\right) \mathbf{H}_{k}+\alpha \mathbf{I}\right]^{-1} \mathbf{H}_{k, l}^{(p) *}
$$

$\left((\cdot)^{H}\right.$ denote the Hermitian matrix), with $\alpha=$ $E\left[\left|N_{k}^{(r)}\right|^{2}\right] / E\left[\left|S_{k, l}^{(p)}\right|^{2}\right]$, common to all $l, r$ and $p$,

$$
\gamma_{l}^{(p)}=\frac{1}{M} \sum_{k=0}^{M-1} \sum_{q=0}^{K-1} \sum_{r=0}^{L_{R}} F_{k+q M, l}^{(p, r)} H_{k+q M, l}^{(p, r)}
$$

and $\quad \mathbf{H}_{k, l}^{(p)} \quad=\quad\left[H_{k, l}^{(p, 1)} \cdots H_{k, l}^{\left(p, L_{R}^{(p)}\right)} \quad \ldots\right.$ $\left.H_{k+(K-1) M, l}^{(p, 1)} \cdots H_{k+(K-1) M, l}^{\left(p, L_{R}^{(p)}\right)}\right]^{T}$.
The optimum feedback coefficients are given by

$$
\mathbf{B}_{k, l}^{(p)}=\mathbf{P}\left(\mathbf{H}_{k} \mathbf{F}_{k, l}^{(p)}-\Gamma_{v(p, l)}\right)
$$

where $\Gamma_{v}$ is a vector with zeros in all positions except the $v$ th and $v(p, l)$ is the position associated to the $l$ th layer of the $p$ th MT, given by

$$
v(p, l)= \begin{cases}l, & p=1 \\ \sum_{p^{\prime}=1}^{p-1} L_{T}^{\left(p^{\prime}\right)}+l, & p>1 .\end{cases}
$$

If we do not have data estimates for the different layers $\rho_{l^{\prime}}^{\left(p^{\prime}\right)}=0\left(p^{\prime}=1,2, \ldots, P ; l^{\prime}=1,2, \ldots, L_{T}^{\left(p^{\prime}\right)}\right)$, and the feedback coefficients are zero. Therefore, (12) reduces to

$$
\tilde{A}_{k, l}^{(p)}=\mathbf{F}_{k}^{T} \mathbf{Y}_{k},
$$

which corresponds to the linear receiver.

It can be shown that the optimum feedforward coefficients can be written in the form

$$
F_{k+q M, l}^{(p, r)}=\sum_{p^{\prime}=1}^{P} \sum_{l^{\prime}=1}^{L_{T}^{\left(p^{\prime}\right)}} H_{k+q M, l^{\prime}}^{\left(p^{\prime}, r\right) *} I_{k, l, l^{\prime}}^{\left(p, p^{\prime}\right)}
$$

( $k=0,1, \ldots, M-1 ; q=0,1, \ldots, K-1)$, with the set of coefficients $\left\{I_{k, l, l^{\prime}}^{\left(p, p^{\prime}\right)} ; p^{\prime}=1, \ldots, P ; l^{\prime}=1,2, \ldots, L_{T}^{\left(p^{\prime}\right)}\right\}$ satisfying the set of $K \cdot L_{T}^{(p)}$ equations

$$
\begin{array}{r}
\sum_{p^{\prime \prime}=1}^{P} \sum_{l^{\prime \prime}=1}^{L_{T}^{\left(p^{\prime \prime}\right)}} I_{k, l, l^{\prime \prime}}^{\left(p, p^{\prime \prime}\right)} . \\
\left(\left(1-\rho_{l^{\prime}}^{\left(p^{\prime}\right) 2}\right) \sum_{q^{\prime}=0}^{K-1} H_{k+q^{\prime} M, l^{\prime \prime}}^{\left(p^{\prime \prime}, r\right) *} H_{k+q^{\prime} M, l^{\prime}}^{\left(p^{\prime}, r\right)}+\alpha \delta_{l^{\prime}, l^{\prime \prime}} \delta_{p^{\prime}, p^{\prime \prime}}\right)= \\
=\delta_{l, l^{\prime}} \delta_{p, p^{\prime}}, \quad p^{\prime}=1,2, \ldots, P ; l^{\prime}=1,2, \ldots, L_{T}^{\left(p^{\prime}\right)} .
\end{array}
$$

The computation of the feedforward coefficients from (23) is simpler than the direct computation, from (18), especially when $N_{L}<K \cdot L_{R}$.

\section{Implementation Issues}

\section{A. Multiple Transmit Antennas vs Multicode Schemes}

Let us assume that the use of a single spreading code with a constellation QPSK (Quaternary Phase Shift Keying) corresponds to the data bit rate $R_{b}$. If we want to duplicate the bit rate while maintaining a QPSK constellation we could assign two spreading codes to a given MT, which corresponds to employing multicode CDMA schemes [14], or we could employ a space multiplexing scheme, where the MT has two antennas, each one transmitting a different data stream (naturally, this means that the BS needs two receive antennas, at least).

The major problem with multicode CDMA schemes is that the envelope fluctuations and PMEPR (Peak-to-Mean Envelope Power Ratio) of the transmitted signal increase with the number of codes that is being assigned to a given MT. 

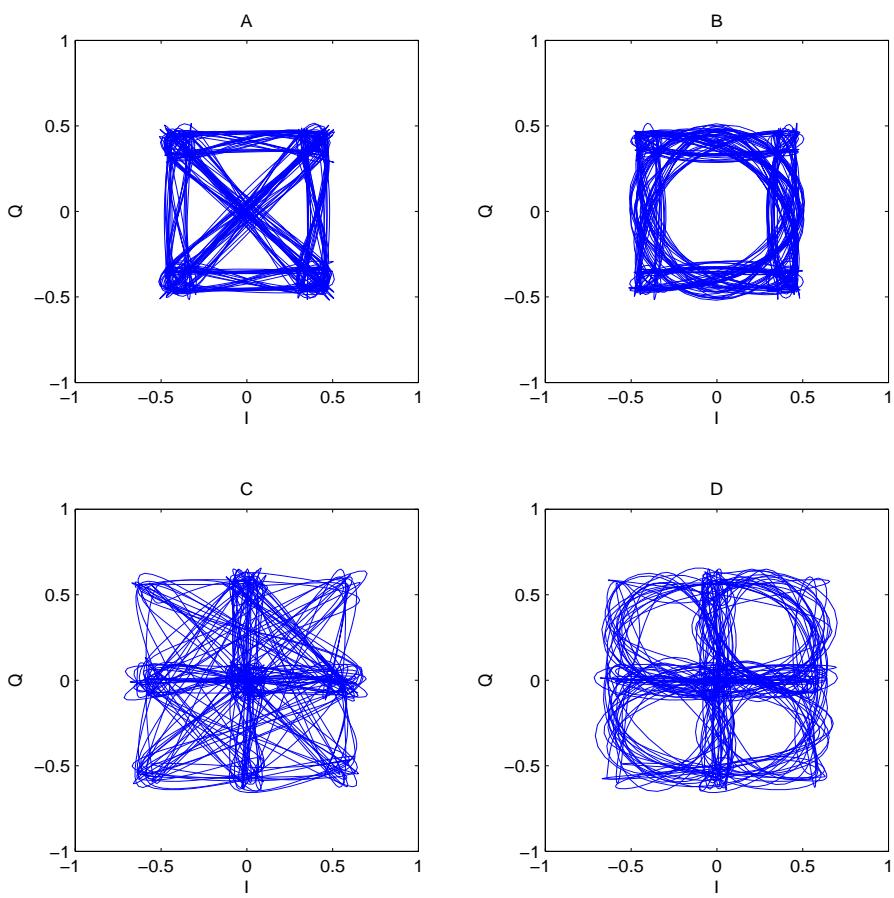

Fig. 3. I-Q diagrams for the following situations: (A) single-code QPSK; (B) single-code OQPSK; (C) multicode QPSK; (D) multicode OQPSK.

For instance, fig. 3 shows the I-Q diagrams of the transmitted signal for a single-antenna MT with one or two spreading codes assigned to it and QPSK constellations (we have $\mathrm{PMEPR}=2.8 \mathrm{~dB}$ for the single-code case and $\mathrm{PMEPR}=5.2 \mathrm{~dB}$ for the multicode case), as well as the corresponding I$\mathrm{Q}$ diagrams for OQPSK schemes (Offset QPSK) (we have $\mathrm{PMEPR}=2.6 \mathrm{~dB}$ for the single-code case and $\mathrm{PMEPR}=5.1 \mathrm{~dB}$ for the multicode case). A square-root raised cosine filtering with roll-off factor 0.5 is assumed. Clearly, the envelope fluctuations are much higher for the multicode scheme. The single-code case with an OQPSK scheme is of particular interest since it is compatible with a low-cost, grossly nonlinear power amplification, especially when MSK-type (Minimum Shift Keying) signals are employed.

By employing a spatial multiplexing schemes with two transmit antennas, we will need two power amplifiers; however, since the signal at the input of each amplifier is a "single-code signal", its envelope fluctuations can be very low, allowing an efficient power amplification. Moreover, the peak power required for each amplifier is lower than for the multicode case. For MTs that require very high bit rates the required number of amplifiers/antennas is also high, which is not feasible to implement. For these situations, it might be better to adopt a multicode scheme with a single amplifier and a single transmit antenna, eventually combined with some suitable signal processing for reducing the envelope fluctuations of the transmitted signals [15], [16].

It should be noted that, the different transmit and receive antennas should be almost uncorrelated. This is not a problem at the BS, since the separation between antennas can be relatively high. However, for a typical MT, which is expected to have small dimensions, this might be a problem. In this case, we could use orthogonal spreading codes for the different antennas.

Our simulations show that we can have essentially the same performance with uncorrelated antennas or highly correlated antennas, with orthogonal spreading codes. In fact, if we have a single MT, our receiver behaves as the one proposed in [10], in the second case.

It should also be noted that the separation between the data streams associated to the different antennas and the different MTs results from the combination of the spreading codes and the corresponding channel frequency responses (see (6)). This means that we have essentially the same performance regardless of the spreading codes, provided that we have severely time-dispersive channels and the corresponding frequence responses are highly uncorrelated.

\section{B. Detection Strategy}

The receiver structure described in the previous section can be regarded as an iterative multiuser detector with interference cancelation. The most common interference cancelation strategies are the PIC (Parallel Interference Cancelation) and the SIC (Successive Interference Cancelation) schemes ${ }^{4}$. For the SIC receiver, we cancel the interference from all the antennas of each MT using the most updated version of it, as well as the residual ISI for the data stream that is being detected. For the PIC receiver, we cancel the interference, as well as the residual ISI, employing the data estimates from the previous iteration. In general, the achievable performance is similar for both schemes, although the convergence is faster for the SIC receiver [12], provided that we detect first the MTs for which the power at the BS is higher. The main advantage of the PIC structure is the possibility of a parallel implementation, with the simultaneous detection of all layers, at each iteration.

The computation of the feedforward coefficients requires solving a system of $L_{R} K$ equations, or a system of $N_{L}$ equations if we use (23)-(24), for each frequency. Whenever a given layer has a very high reliability $\left(\rho_{l}^{(p)} \approx 1\right)$, we can remove its interference almost entirely. This means that we can ignore that layer when detecting the others; therefore the computation of the feedforward coefficients requires solving a system with a smaller dimension, provided that we use (23)(24).

\section{Performance Results}

In this section, we present a set of performance results concerning the receiver structure described here for the uplink of a CP-assisted DS-CDMA system employing spatial multiplexing. The spreading factor is $K=4$ and the BS has $L_{R}=2$ receive antennas. We consider a fully loaded scenario with $P=4 \mathrm{MTs}$, each one with $L_{T}^{(p)}=2$ transmit antennas

\footnotetext{
${ }^{4}$ It should be noted that our SIC and PIC receivers are iterative in the sense that each user is estimated several times, while some papers define a SIC receiver where each user/layer is estimated only once.
} 

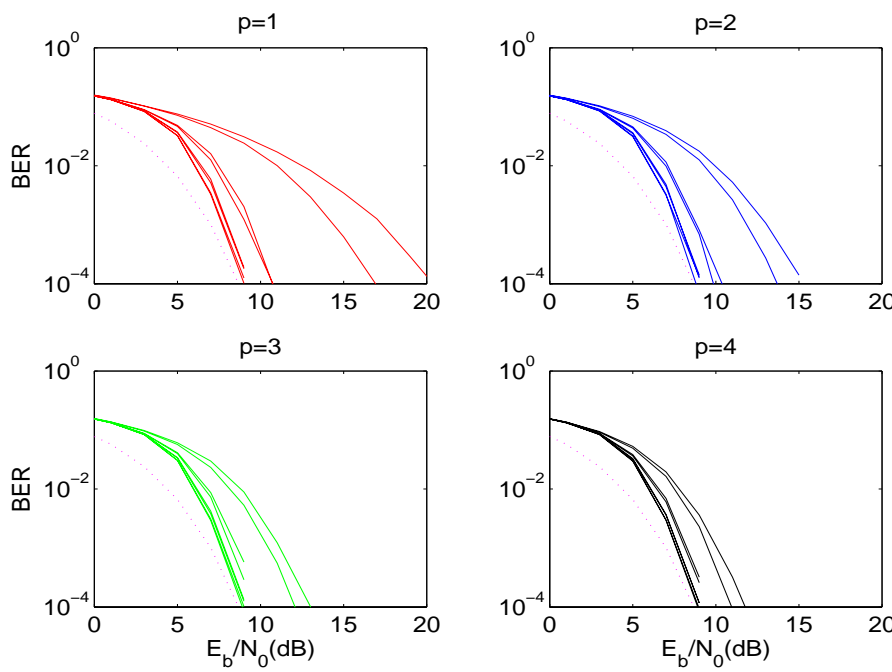

Fig. 4. BER performances for layer $l(l=1,2)$ and iteration $i=1,2,3,4$ (solid lines) when the average received power is identical for all MTs (layers detected latter have better performances, i.e., the worse performance is for $(i, l)=(1,1)$, followed by $(1,2),(2,1),(2,2),(3,1),(3,2),(4,1)$ and $(4,2))$, together with the corresponding MFB (dotted lines).

(i.e., a spatial multiplexing scheme with two layers per MT). We have $M=64$ data symbols for each layer, corresponding to blocks with length $N=K M=256$, plus an appropriate cyclic extension. QPSK constellations, with Gray mapping, are employed. We have a severely time-dispersive channel and uncorrelated antennas at the BS and at each MT. We consider uncoded BER performances under perfect synchronization and channel estimation conditions. The power amplifiers at each MT are assumed to be linear.

Let us first assume that the signals associated to each antenna of each MT have the same average power at the receiver (i.e., the BS), which corresponds to a scenario where an "ideal average power control" is implemented. Fig. 4 shows the impact of the number of iterations on the BER for each layer of each MT. For the sake of comparisons, we also include the corresponding single-user MFB performance (Matched Filter Bound). Fig. 5 show the average BER for each MT (i.e., the average over the two transmitted layers). From these figures, we can observe that our iterative receiver structure allows a significant improvement on the BER performance. For a given iteration, the layers that are detected first face stronger interference levels and have worse BER. This is especially important at the first iteration. After four iterations the performances are already similar for all layers, and very close to the MFB.

Let us assume now that we have different average receive powers for the different MTs. We will assume that the difference between the average receive power of MT 1 and the average receive power of MTs 2,3 and 4 are $3 \mathrm{~dB}, 6 \mathrm{~dB}$ and $9 \mathrm{~dB}$, respectively. Clearly, the MTs with higher $p$ face stronger interference levels. The corresponding layer' performances are depicted in fig. 6 . Once again, the proposed iterative receiver

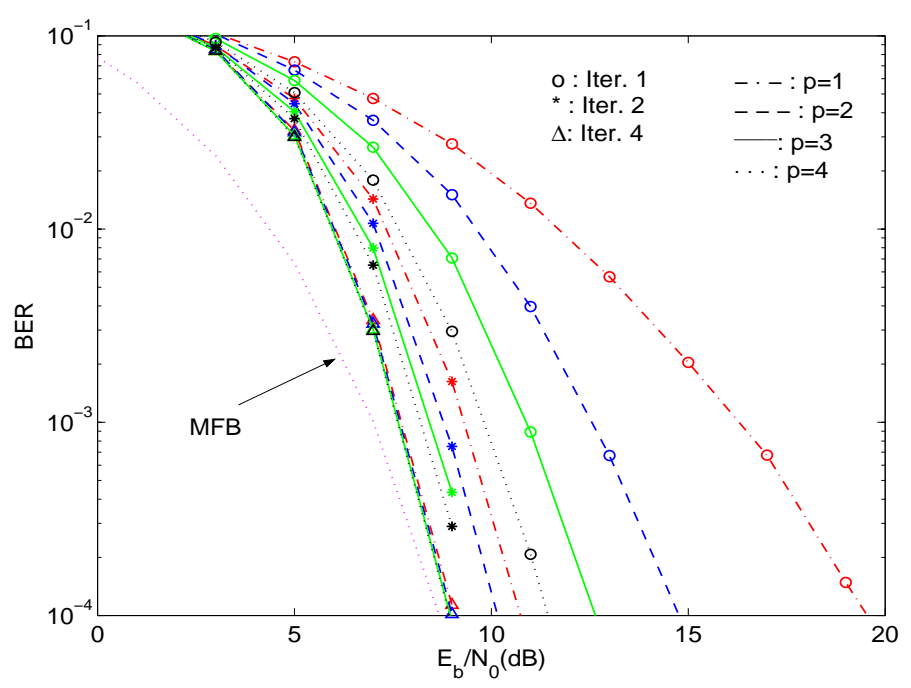

Fig. 5. Average BER performance for each MT and 1, 2 or 4 iterations, when the average received power is identical for all MTs.
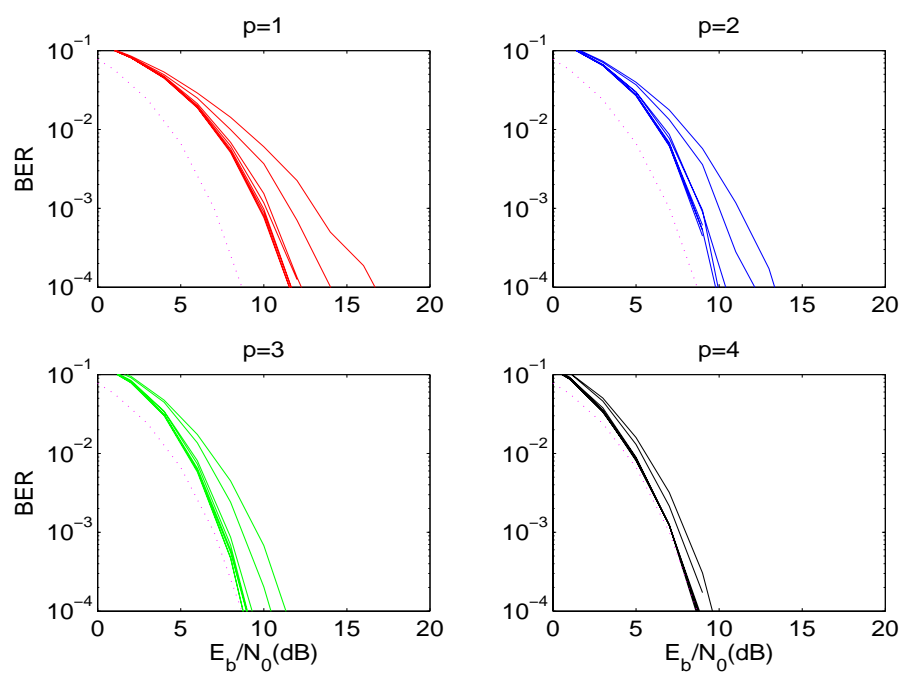

Fig. 6. As in fig 4, but with different average received powers for different MTs.

allows significant performance gains. The performance of MTs with lower power asymptotically approaches the MFB when we increase the number of iterations; however, for MTs with higher power, the BER at $10^{-4}$ is still between 1 or $2 \mathrm{~dB}$ from the MFB. This can be explained from the fact that the BER is much lower for high-power users, allowing an almost perfect interference cancelation of their effects on low-power users; therefore, the corresponding performances can be very close to the MFB. The higher BERs for the low-power users preclude an appropriate interference cancelation when we detect highpower users (see also fig. 7).

\section{Conclusions}

In this paper we considered the uplink transmission within a DS-CDMA system employing CP-assisted block transmission 


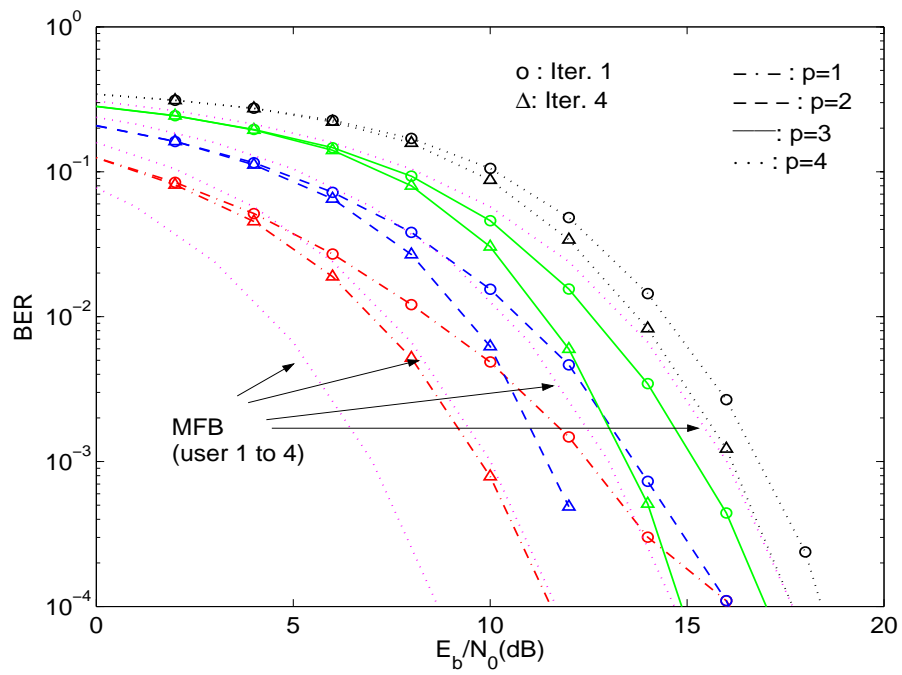

Fig. 7. Average BER performance for each MT, expressed as a function of the $E_{b} / N_{0}$ of the first MT.

techniques combined with spatial multiplexing techniques that require multiple antennas at both the transmitter and the receiver. We presented an efficient frequency-domain receiver structure with an iterative MUD.

It was shown that the performance of the proposed receiver can be close to the single-user MFB, even for fully loaded systems, in severely time-dispersive channels and/or in the presence of strong interfering signals.

\section{References}

[1] G. J. Foschini and M. J. Gans, "On limits of Wireless Communications in Fading Enviroments when Using Multiple Antennas," Wireless Personal Communications, Vol. 6, pp. 315-335, March 1998.

[2] L.Cimini Jr., "Analysis and Simulation of a Digital Mobile Channel using Orthogonal Frequency Division Multiplexing", IEEE Trans. on Commun., Vol. 33, pp. 400-411, July 1985.

[3] H.Sari, G.Karam and I.Jeanclaude, "An Analysis of Orthogonal Frequency-division Multiplexing for Mobile Radio Applications", In Proc. IEEE Vehic. Tech. Conf., VTC'94, pp. 1635-1639, Stockholm, June 1994.

[4] A. Gusmão, R. Dinis, J. Conceição, and N. Esteves, "Comparison of Two Modulation Choices for Broadband Wireless Communications”, IEEE VTC'00 (Spring), Tokyo, Japan, May 2000.

[5] D.Falconer, S.Ariyavisitakul, A.Benyamin-Seeyar and B.Eidson, "Frequency Domain Equalization for Single-Carrier Broadband Wireless Systems", IEEE Comm. Mag., Vol. 4, No. 4, pp. 58-66, April 2002.

[6] A. Viterbi, CDMA: Principles of SS Communication, Addison Wesley, 1995.

[7] K. Baum, T. Thomas, F. Vook, V. Nangia, "Cyclic-Prefix CDMA: An Improved Transmission Method for Broadband DS-CDMA Cellular Systems", IEEE WCNC, pp. 183-188, 2002.

[8] N. Benvenuto and S. Tomasin, "Block Iterative DFE for Single Carrier Modulation", IEE Elec. Let., Vol. 39, No. 19, pp. 1144 1145, Sep. 2002.
[9] N. Benvenuto and S. Tomasin, "Iterative Design and Detection of a DFE in the Frequency Domain", IEEE Trans. on Comm., Vol. 53, No. 11, pp. 1867-1875, Nov. 2005.

[10] P. Silva and R. Dinis, "An Iterative Frequency-Domain Decision Feedback Receiver for CDMA Systems”, , ISWCS'04, Sep. 2004.

[11] W. Gardner, "Exploitation of Spectral Redundancy in Cyclostationary Signals", IEEE Signal Processing Mag., Vol. 9, Aug. 1991.

[12] P. Silva and R. Dinis, "SIC and PIC Multiuser Detection for Prefix-Assisted DS-CDMA Systems”, , WOC'05, July. 2005.

[13] R. Dinis, A. Gusmão, and N. Esteves, "On Broadband Block Transmission over Strongly Frequency-Selective Fading Channels", Proc. Wireless 2003, Calgary, Canada, July 2003.

[14] N.Guo and L.Milstein, Uplink Performance Evaluation of Multicode DS/CDMA Systems in the Presence of Nonlinear Distortions, IEEE J. on Sel. Areas in Comm., Vol. 18, No. 8, pp. 1418-1428, Aug. 2000.

[15] O.Väänänen, J.Vankka, T.Viero and K.Halonen, "Reducing the Crest Factor of a CDMA Downlink Signal by Adding Unused Channelization Codes", IEEE Comm. Letters, Vol. 6, No. 10, pp. 443-445, Oct. 2002.

[16] R.Dinis and A.Palhau, "A Class of Signal Processing Schemes for Reducing the Envelope Fluctuations of CDMA Signals", IEEE Trans. on Comm., May 2005. 